\title{
OpenPMU Technology Platform for Synchrophasor Research Applications
}

\author{
David M. Laverty, Member, IEEE, Luigi Vanfretti, Member, IEEE, Robert J. Best, \\ D. John Morrow, Member, IEEE, Lars Nordstrom, Member, IEEE, Moustafa Chenine, Member, IEEE
}

\begin{abstract}
OpenPMU, the Open Source Phasor Measurement Unit, is a platform for the development of Synchrophasor measurement technology in an open source manner. The OpenPMU design modularizes the key components of a Phasor Measurement Unit (PMU) into three subsystems; measurement, phase estimation and telecoms. The advantage to the researcher is that the interfaces between each subsystem are open and human readable, allowing for rapid integration of new work into an existing framework. This allows for new work to be prototyped rapidly and tested in an online environment.

This paper introduces the OpenPMU technology platform. The subsystem modules and the methods of exchanging data are described. Present work on the platform is discussed and an open invitation to join the development process is extended to all.
\end{abstract}

Index Terms-Phasor Measurement Unit, Open Source, Synchrophasor, Smart Grid

\section{INTRODUCTION}

$S^{s}$ ynchrophasor measurement is increasingly becoming a standard tool of the electrical power systems engineer. Access to time synchronized measurements, especially phase angle, opens up a suite of new applications and is attracting interest amongst both the research and utility communities alike. It affords the means to improve understanding and modeling of power system phenomena, and can be directly applied in power system protection schemes. Vendors of various measurement devices are beginning to offer Synchrophasor measurement as a secondary function on their devices as a marketing incentive, indicating that the technology is now incredibly cost effective and will become increasingly ubiquitous.

The authors have interests in the application of Synchrophasors to anti-islanding protection [1] and to the phase control of islanded dispatchable generation, "Synchronous Islanding" [2]. In developing these technologies, the authors found it necessary to develop their own Phasor Measurement Unit (PMU) to meet the

Invited Paper submitted for the IEEE PES GM Panel Session on "Open Source Software enabling the Smart Grid"

D. M. Laverty, R. Best and D. J. Morrow are with the Electrical Power and Energy Research Cluster, Queen's University Belfast, Ashby Building, Belfast, BT9 5AH, Northern Ireland (e-mail: david@laverty.org.uk; r.best@qub.ac.uk,dj.morrow@ee.qub.ac.uk).

L. Vanfretti, L. Nordstrom and M. Chenine are with the Royal Institute of Technology, KTH, Stockholm, Sweden (e-mail: luigiv@kth.se, larsn@ics.kth.se, moustafa.chenine@ics.kth.se). performance they required. Difficulties encountered in the development of this system included the design of a GPS disciplined data acquisition device, and lack of a readily available tool set to communicate in the IEEE C37.118 Synchrophasor representation format. Another consideration encountered was the lack of a defined standard for the transient performance of PMU devices [3].

The authors PMU device was developed from first principles and generally in accordance to the method proposed by Phadke in [4]. The authors have subsequently found that several other researchers have embarked, sometimes simultaneously, on developing their own PMUs, e.g. the "DTU PMU" [5] and "Grid Trak" [6]. The authors consider that each of these PMU systems is a great accomplishment, but that there is needless duplication of effort in these designs. Thus the authors propose that an open source platform on which to develop such technology would benefit the research community.

This paper explains the objective behind open sourcing the PMU design, the architecture and information exchange in the technology platform, describes the subsystems of the PMU, licensing models adopted, programming language and means by which to become involved in the project.

\section{WHY OPEN SOURCE}

The first question that many people ask when they hear of the OpenPMU project is, "Why open source?" Reasons that are given for open source software include giving total control of the software over to the end user, thereby empowering them to adapt and tailor it to their specific application. Other reasons include the opinion that many people scrutinizing the code leads to a more secure product as a result of errors being identified, fixed and returned to the community. Red Hat says, "Freedom means choice. Choice means power. That's why we believe open source is inevitable. It returns control to the customer. You can see the code, change it, learn from it. Bugs are found and fixed quickly. [7]"

The ideals of open source software are admirable, but many smaller or niche projects fall short of achieving those aims due to a lack of community involvement. The authors believe that the community of researchers interested in PMU technology is now sufficiently established to be able to sustain an open source project, and what's more, encourage its growth and development.

The present partners in the OpenPMU project may be viewed online at the projects homepage on 
www.OpenPMU.org [8]. The project is primarily run and maintained by universities, which benefit greatly from the scope for international collaboration.

The objective of OpenPMU is to work towards developing a fully featured PMU compliant with the IEEE Std. C37.118 [9] which defines the measurement aspects, performance and interfacing of Synchrophasor equipment. While this is the ultimate goal, initially development will concentrate on creating a technology platform for development of the individual componenets of the system. OpenPMU is not intended as competition to commercial device manufacturers, rather it is desired to be a tool to compliment commercial devices in the research environment.

\section{OPENPMU TECHNOLOGY PLATFORM}

The authors have been actively working on open sourcing their current PMU system for over a year. In so doing it has become apparent that open sourcing a PMU as a complete system is disadvantageous, since a change to one component of the system necessitates adjustments to all other components in the system. What is much more preferable is a platform in which the major subsystems are separate and the interface between the subsystems is defined.

The authors propose that there are three major elements to a PMU device capable of generating real-time Synchrophasor data. These are the measurement subsystem, the phase estimation subsystem, and the telecommunications subsystem, shown in Figure 1. Other optional subsystems that compliment basic PMU functionality include disturbance recording and local storage of data. Since they are not core subsystems, they are not described in this paper.

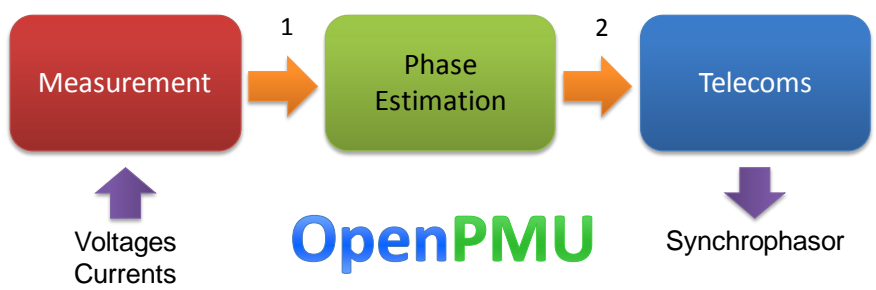

Fig. 1. Subsystems of the OpenPMU platform

The subsystem and their interfaces are now described in the following sections of this paper.

\section{A. Measurement Subsystem}

The measurement subsystem's purpose is to provide time coded sample data to the phase estimation stage. The data acquisition process itself must be disciplined to an external time signal, typical UTC derived from the GPS global navigation satellite system.

It is typical in PMU systems to sample the data such that there are 128 samples per cycle $\left(2^{7}\right)$. On $50 \mathrm{~Hz}$ and $60 \mathrm{~Hz}$ systems this yields a sampling rate of $6.4 \mathrm{kHz}$ and $7.68 \mathrm{kHz}$ respectively. Some PMU systems sample at rates of 256 or 512 samples per cycle. Sampling resolution is typically 16-bits or less. These are not high metrics for an Analog-toDigital converter (ADC) but the difficulty lies in synchronizing the sampling with the GPS time signal.
Consequently it is necessary to use parallel ADCs controlled by a high speed microprocessor or perhaps an FPGA. Various architectures are employed in different commercial products. The authors have developed a system which uses a simple PIC microcontroller to discipline an undisciplined National Instruments data acquisition card, described in [10] and designs available on the OpenPMU project web pages.

\section{1) Expression of Measurement Data}

The data from the measurement subsystem should be pushed from the measurement device regardless of whether a phase estimation system exists to process the data. The measurement subsystem is a hardware device that will need to communicate with either a PC or an embedded microprocessor which is performing the phase estimation and telecommunications. The authors believe that the best means of making the interface technology agnostic is to use UDP/IP as a protocol and standard Ethernet as the physical media. The data payload itself shall be represented in XML in a format described by the exemplar in Figure 2.

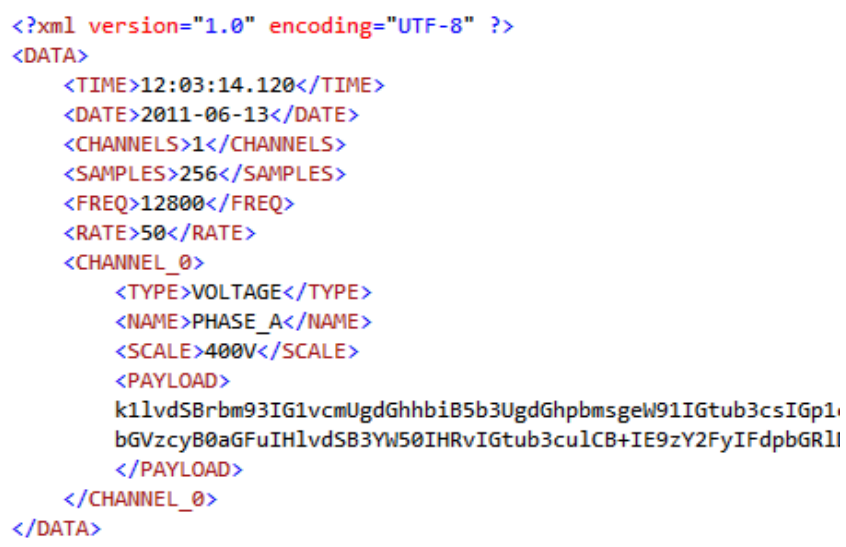

Fig. 2. Exemplar of XML format from measurement subsystem.

$\mathrm{XML}$ is the preferred data representation format since it is human readable. This makes it a more portable format between different development environments where the phase estimation algorithms are to be developed before contribution to the OpenPMU repository, e.g. Matlab, Labview or Python.

Since XML itself is quite bit inefficient in transferring data, due to the high overhead of the individual element tags and limitations of using ASCII, the sample data payload is converted from binary and coded in Base64 representation [11]. At the receiving end, the original sample data can be decoded from Base64.

There are several advantages to using UDP/IP as a transport mechanism for the measurement subsystem. Firstly, the measurement hardware does not need to be located beside the device performing the phase estimation. It may be placed on a long run of Ethernet cable or even routed over the local area network. Several devices may be used simultaneously to acquire data from multiple circuits. A virtual measurement device can be created in software, and through use of the localhost address, used to simulate historical disturbances as online events to test the performance of phase estimation algorithms. The authors are presently developing a virtual measurement subsystem program with this aim. 


\section{B. Phase Estimation}

The phase estimation subsystem might be considered the main component of the PMU software, since this is the subsystem that makes the conversion from raw sample data to a meaningful Synchrophasor. The Synchrophasor describes the amplitude, frequency and phase angle of the sampled waveform. IEEE C37.118 specifies the convention for the representation of Synchrophasors, as depicted in Figure 3.

The phase estimation algorithm might take one of many forms, for example Newton-type algorithm [12], WeightedLeast-Squares / Adaptive Finite Impulse Response (FIR) [13] or dynamic types that vary the measurement algorithm depending on input conditions [14]. The author's field of research is not expert in phase estimation algorithms so at present the OpenPMU software uses a simple FFT / Spectral Leakage type algorithm which acts upon $40 \mathrm{~ms}$ worth of samples windowed by a "Blackman-Harris" function.

It is hoped that experts in the phase estimation community will contribute more sophisticated algorithms to the project.
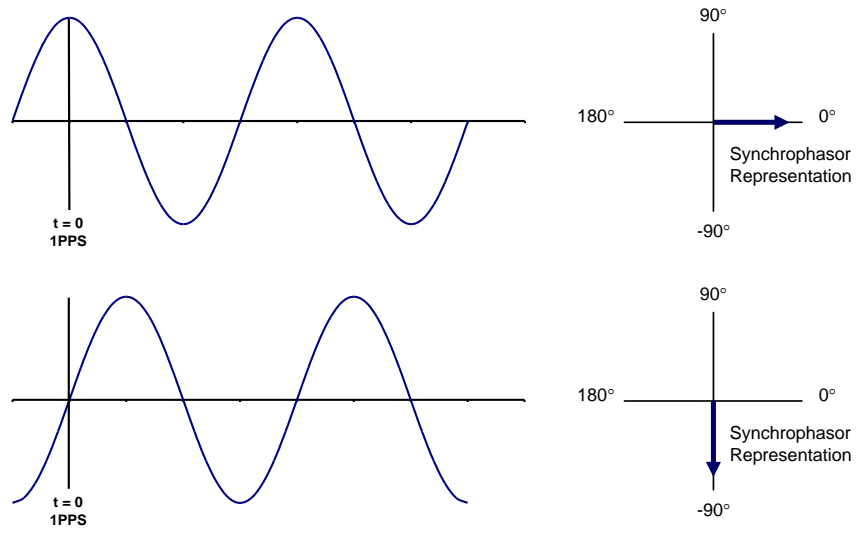

Fig. 3. Synchrophasor representation (cosine convention) [6].

\section{1) Expression of Phase Estimation Data}

The output waveform parameters from the phase estimation subsystem are to be pushed to the telecommunications subsystem regardless as to whether the telecoms subsystem is present. Similar to the measurement subsystem, UDP/IP transport carrying an XML payload is the delivery mechanism. The 'localhost' loopback address allows data to be exchanged between the two modules when they coexist on the same operating system.

At minimum, the phase estimation payload should contain the amplitude, frequency and phase angle for each waveform that is estimated, including the label identifying the source of the data via the measurement subsystem. The payload must be time coded as per the data from the measurement subsystem. When delivered to the telecommunications subsystem, sufficient data must be present so as to complete an IEEE C37.118 Synchrophasor frame.

\section{Telecommunications}

The telecommunications subsystem receives data from the phase estimation stage and produces data that meets the IEEE C37.118 specification for the format of Synchrophasor data. It is essential that the Synchrophasor be standards compliant so as the OpenPMU can interface with data concentrators, such as openPDC [15].

The IEEE standard defines more than the format of the Synchrophasor. It also includes configuration instructions for the PMU device, and the function to start and stop the PMU data stream. This must also be implemented.

Additionally, a web interface should be provided that yields information on the condition of the OpenPMU system and allows configuration of the various modules. Thus a method of configuration data exchange needs to exist between the various components. The authors have yet to establish an exact mechanism, but propose the further use of XML with standardized UDP listening ports on each module to receive configuration instructions. For instance, the measurement subsystem may have options to set the sampling rate, change the resolution, label the channels or give the device a unique ID in a multi measurement device setup.

\section{LICENSING}

\section{A. Software Licensing}

The OpenPMU technology platform, that is to say the interfaces and framework that comprise the three separate subsystems, is currently licensed under the BSD license [16]. Individual modules may be licensed under GPLv3 [17] at their individual contributor's discretion. The present collaborators are currently discussing moving the entire package over to the GPLv3 license in order to simplify licensing the various components. Advantages of GPLv3 include that modifications to the software must be returned to the community, so the original contributor will benefit. Disadvantages include potentially limiting contributions from those who would prefer to be able to make modifications privately without supplying updates to the community. The authors welcome input in this decision making process.

\section{B. Hardware Licensing}

In the event that collaborators develop an OpenPMU hardware measurement system, it will be necessary to have a means of protecting and open sourcing this contribution. The problem arises that open hardware licenses protect the design documentation and instructions, but not the actual hardware itself. Hardware rights are traditionally protected by patents, and as yet the open source community has not found an effective way of replacing patents [18]. [18] even goes as far as to say there are "no good open hardware licenses to date". Of the Open Hardware licenses available, the most complete would seem to be the TAPR Open Hardware License (OHL) [19]. This offers protection on the design rights, allowing products to be made from the designs so long as the designs are distributed with the product. This would seem to fit with the philosophy of the OpenPMU project.

Fortunately the firmware running a measurement subsystem can be considered software and may be protected and licensed under any of the OSS licenses available. 


\section{LANGUAGE}

Choosing a programming language for a large project such as OpenPMU is often a difficult decision. There are many factors to consider; the authors' own experience and background in programming, what the project intends to achieve, and the accessibility to the language by other contributors.

Python is an open source, high level, general purpose programming language. The design of the language ensures that Python code is highly readable and is thus easily maintainable in collaborative projects. Python contains many high level data structures, such as native data types for lists, maps and sets. It is also dynamically typed, which significantly reduces the amount of code required. These features mean that Python developers can be more productive than traditional languages (e.g. C, $\mathrm{C}++$, Java) as they are able focus more on algorithms and problem solving instead of writing large amounts of 'boiler plate' code.

The popularity and uptake of Python has increased rapidly over the last decade and boasts some very high profile commercial users, such as Google, who make heavy use of Python in their web based products, and employ the creator of Python. The recent increase in Python usage is also due to the increasingly large array of high quality third party libraries. Python is a truly general purpose language, with applications in scripting, web programming, desktop applications and scientific computing.

Python may be used on many operating systems, including Microsoft Windows, Linux, Unix, BSD and others. It is attractive that software development may begin in the Windows environment, say, and subsequently find application with very little modification in an embedded microprocessor.

In the scientific computing domain, Python is especially useful due to the availability of the powerful NumPy and SciPy libraries, which provide users with a high level interface for dealing with scientific data and algorithms, without major sacrifices in execution speed. The combination of Python, NumPy and SciPy provides a fully open source and crossplatform development environment for OpenPMU.

\section{OPENPMU ON LABVIEW}

The first generation OpenPMU hardware operates on the Labview platform from National Instruments and is described in [10]. This system can be built at very low cost as is an ideal introduction to Synchrophasor technology for new research students, undergraduate laboratories and even small scale experiments. Since it uses mainly standard laboratory components, it is relatively inexpensive to build a unit.

The Royal Institute of Technology, KTH, Stockholm has successfully constructed a replica of the original Queen's University Belfast unit using the design drawings available on the OpenPMU project web pages. KTH have added support for IEEE C37.118 messaging based on the existing open source phasor protocol library provided by the openPDC [15] project. This adds both UDP and TCP telecoms support for phasor data concentrators (PDCs) and other applications. A screenshot of the Labview OpenPMU is shown in Figure 4.

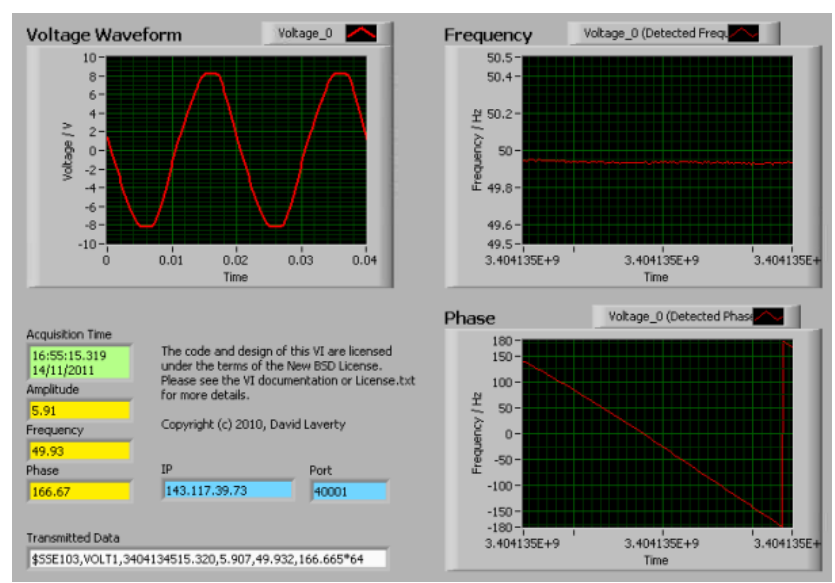

Fig. 4. OpenPMU running in the Labview environment.

\section{COMMunity InVOLVEMENT}

\section{A. Use with Commercial PMU Equipment}

The authors have discussed the possibility of commercial vendors offering options on their products to provide time coded sample data for use with the OpenPMU software. Although this necessitates the purchase of a PMU device, this offers the advantage of being able to use a fully certified piece of equipment in an online environment, which may be the only option available to acquire data from utility sites and substations. Talks are in the early stages and offers from commercial PMU vendors to offer such functionality are warmly welcomed.

\section{B. Low Cost PMU for Low Voltage Applications}

OpenPMU developed out of the need for a low cost PMU, with transparent operation, for use in applications on low voltage networks were the expense of current commercial PMUs could not be justified. Functions such as disturbance / transient event recorder are not necessary for many of these applications.

The authors are presently pursuing the development of a low cost measurement subsystem that meets the specification of the OpenPMU project. This subsystem, coupled with a microprocessor capable of running the phase estimation and telecommunications modules would offer a very low cost solution for prototyping low voltage applications and could form the basis of future projects. Contributions to this discussion and collaborators are very welcome.

\section{Getting Involved}

To get involved with the OpenPMU project and to communicate with the authors of this paper, please visit www.OpenPMU.org. The project is hosted on the Codeplex system, which provides Wiki style documentation, message boards and news feeds. For less formal contact and announcements, the authors maintain a Facebook page. Simply search for 'OpenPMU' within Facebook.

The authors particularly welcome suggestions for improving the project, and welcome partners interested in contributing to the project. Please do not hesitate to make contact if you have any questions regarding taking part in OpenPMU or building your own OpenPMU. 


\section{REFERENCES}

[1] Laverty, D.M.; Morrow, D.J.; Littler, T.; Crossley, P.A., "Loss-of-Mains Detection by Internet Based ROCOF," Developments in Power System Protection, 2008. DPSP 2008. IET 9th International Conference on, pp.263 268, 17-20 March 2008

[2] Best, R.J.; Morrow, D.J.; Laverty, D.M.; Crossley, P.A.; , "Synchrophasor Broadcast Over Internet Protocol for Distributed Generator Synchronization," Power Delivery, IEEE Transactions on, vol.25, no.4, pp.2835-2841, Oct. 2010

[3] Laverty, D.M.; Morrow, D.J.; Best, R.; Crossley, P.A.; , "Performance of phasor measurement units for wide area real-time control," Power \& Energy Society General Meeting, 2009. PES '09. IEEE , vol., no., pp.15, 26-30 July 2009

[4] Phadke, A.G.; Thorp, J.S., "History and Applications of Phasor Measurements," Power Systems Conference and Exposition, 2006. PSCE '06. 2006 IEEE PES, pp.331-335, 2006

[5] Design, installation and application of Phase Measuring Units (PMUs), DTU, 2009

Internet: http://power.inescporto.pt/EES-UETP/DTU_PMU_Course.pdf

[6] GridTrak Power Grid Frequency Monitor, Arnold Stadlin,

Internet: http://www.phzmonitor.com/

[7] Red Hat, "Why Open Source?",

Internet: http://www.redhat.com/about/whyopensource/

[8] OpenPMU, Open Source Phasor Measurement Unit, Internet: http://www.openpmu.org/

[9] "IEEE Standard for Synchrophasors for Power Systems," IEEE Std C37.118 2005 (Revision of IEEE Std 1344-1995), 2006

[10] Laverty, D.M.; Morrow, D.J.; McKinley, A.; Cregan, M.; , "OpenPMU: Open source platform for Synchrophasor applications and research," Power and Energy Society General Meeting, 2011 IEEE, vol., no., pp.16, 24-29 July 2011

[11] The Internet Society, Base64 Encoding, 2006 Internet: http://tools.ietf.org/html/rfc4648

[12] Terzija, V.; Stanojevic, V., "Power quality indicators estimation using robust Newton-type algorithm," Generation, Transmission and Distribution, IEE Proceedings- , vol.151, no.4, pp. 477-485, 11 July 2004

[13] Kusljevic, M.D.; Tomic, J.J.; Jovanovic, L.D.; , "Frequency Estimation of Three-Phase Power System Using Weighted-Least-Square Algorithm and Adaptive FIR Filtering," Instrumentation and Measurement, IEEE Transactions on, vol.59, no.2, pp.322-329, Feb. 2010

[14] Warichet, J.; Sezi, T.; Maun, J.C.: "A Synchrophasor Measurement Algorithm Suitable for Dynamic Applications," $16^{\text {th }}$ Power Systems Computation Conference, PSCC 2008

[15] OpenPDC Project Pages, Codeplex

Internet: http://openpdc.codeplex.com/

[16] The Open Source Initiative OSI - The BSD License

Internet: http://www.opensource.org/licenses/bsd-license.php

[17] GNU General Public License

Internet: http://www.opensource.org/licenses/bsd-license.php

[18] Open Hardware Licenses, The Foundation for P2P Alternatives Internet: http://p2pfoundation.net/Open_Hardware_Licenses

[19] The TAPR Open Hardware License,

Internet: http://www.tapr.org/ohl.html

\section{BIOGRAPHIES}

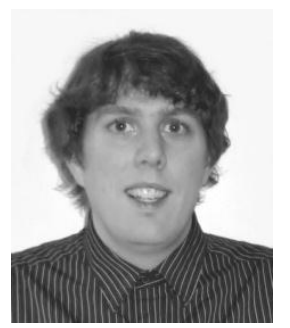

David M. Laverty (S'07, M'10) was born in Belfast, Northern Ireland, in 1984. He received the M.Eng and Ph.D. degrees from Queen's University Belfast, Belfast, UK, in 2006 and 2010 respectively. His placements as an undergraduate were with Northern Ireland Electricity and Universität Paderborn, Germany.

Since 2011 he has been a Lecturer with the Electrical Power and Energy Research Cluster at Queen's University Belfast, Belfast, UK. His current research interests are in power system measurements, anti-islanding detection, phasor measurements, and Smart Grid telecommunications, messaging and security.

Dr. Laverty is a member of the IEEE and a member of and volunteers with the Institution of Engineering and Technology (IET).

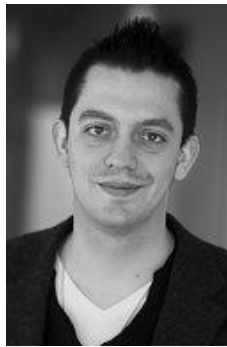

Luigi Vanfretti (M'10) is an Assistant Professor at the Electric Power Systems Division, School of Electrical Engineering, KTH Royal Institute of Technology, Stockholm, Sweden. He received his M.Sc. and Ph.D. in 2007 and 2009, respectively, both in Electric Power Engineering from Rensselaer Polytechnic Institute, Troy, NY, USA. His main research interest is on the development of PMU data-based applications. Since 2009, he has served as Secretary of the IEEE PES CAMS Task Force on Open Source. $\mathrm{He}$ is an evangelist of Free/Libre and Open Source Software.

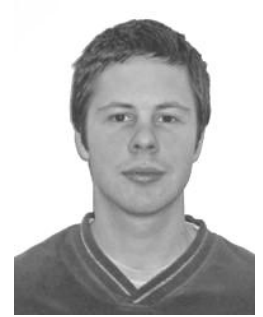

Robert J. Best was born in Belfast, Northern Ireland, in 1980. He received the M.Eng. and Ph.D. degrees from Queen's University Belfast, Belfast, U.K., in 2004 and 2008, respectively.

$\mathrm{He}$ is currently a Research Fellow with the Electrical Power and Energy Research Cluster at Queen's University Belfast, with research interests in power system islanding, distributed generation and electric machinery.

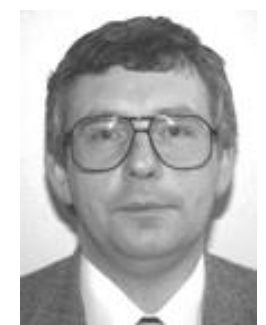

D. John Morrow (M'99) was born in Dungannon, Northern Ireland, in 1959. He received the B.Sc and $\mathrm{Ph} . \mathrm{D}$. degrees from Queen's University Belfast, Belfast, U.K., in 1982 and 1987, respectively.

Since 1987, he has been a Lecturer in electrical engineering at Queen's University Belfast, Belfast, UK with research and consulting interests in electric power systems, power system instrumentation, and gen-set controllers.

Dr. Morrow is a member of the Institute of Engineering and Technology and also a member of the IEEE PES Excitation Systems Subcommittee working group since 1999.

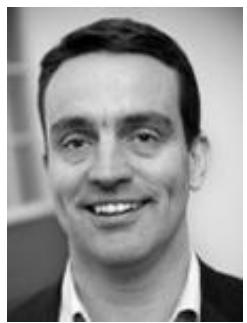

Lars Nordstrom (M'06) received the M.Sc. degree in electrical engineering and the Ph.D. degree in industrial control systems, both from $\mathrm{KTH}-\mathrm{The}$ Royal Institute of Technology, Stockholm, Sweden. $\mathrm{He}$ is an Associate Professor in Power System Management and Director of the Swedish Centre of Excellence in Electric Power Engineering, an industry-university research center, located at $\mathrm{KTH}$. His area of research is power systems management and related information exchange, including application of decision theory on information system architectures and the application of ICT to power system problems. Dr. Nordstr" om is chairman of IEC TC57's Swedish committee, and a member of the IEC's Strategic group on Smart grids as well as National Cigre SC D2 representative for Sweden.

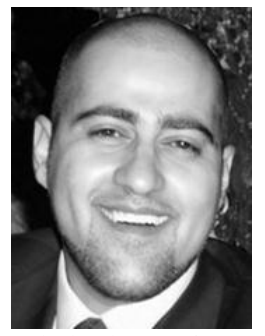

Moustafa Chenine (Student Member '07) received his B.Sc. with a major in Computer Information Systems from The American College of Greece in 2004. He then joined KTH - The Royal Institute of Technology where he worked towards his M.Sc. (2006) in Information Systems and Licentiate of Engineering in Electrical Engineering (2009). He is a $\mathrm{PhD}$ Student at the department of Industrial Information and Control Systems at $\mathrm{KTH}$, doing research in the field of ICT Infrastructure optimizations for Smart Grid systems, specifically focusing on interoperability, security and performance aspects of Wide Area Monitoring and Con trol Systems. 